ISSN 1112-9867

Available online at http://www.jfas.info

\title{
ROLLING BEARING MONITORING ALGORITHM BY WAVELET SCALING COMPARISON
}

\author{
S. E. Rinatovich ${ }^{1, *}$, V. Y. Vitalevich ${ }^{2}$, A. M. Victorovich ${ }^{2}$, I. E. Vyacheslavovna ${ }^{2}$ \\ ${ }^{1}$ Kazan Federal University \\ ${ }^{2}$ Kazan State Power Engineering University
}

Published online: 08 August 2017

\begin{abstract}
A rolling bearing is the most common element of any rotary mechanism design and, at the same time, the most vulnerable element that determines the operability and the durability of equipment. The purpose of the work is to develop the method of bearing condition control by vibration parameters on the basis of scale graph comparison of a continuous wavelet analysis. The goal is achieved by the solution of the following tasks: to determine informative criteria that allow to detect the bearing defects by the analysis of its vibration; to develop the algorithm of bearing defect detection by the parameters of their vibrations, which make it possible to control products in automatic mode.

They performed the simulation of whole and defective rolling bearing signals by the synthesis of frequency components characteristic for defects.

The correlation coefficient of spectra and the rank correlation of Spearman's spectra were used in the work for spectrum comparison. The comparison of the scaling graphs obtained by continuous wavelet transformation was carried out using PSNR metric.

The approach is suggested in order to separate the bearings into "defect-free" or "defective" ones. This approach is typical for the procedures of anomaly rejection. The analysis algorithm interprets the set of calculated values of some statistics (p1, p2, .., pm) as a set of measured values of an abstract parameter and applies the following procedure to this set: the position
\end{abstract}

Author Correspondence, e-mail: mr.emilsr@gmail.com

doi: http://dx.doi.org/10.4314/jfas.v9i2s.71 
estimate $\mathrm{p}$ is calculated; the variance estimate $\mathrm{S}$ is calculated as the median of absolute deviations with respect to a position estimate; a confidence interval is developed for a given level of significance $\alpha$. The bearings with comparison results that fall within the confidence interval are considered defect-free ones, and the ones that didn't fall within this interval are considered as defective.

Keywords: rolling bearings, defects, nondestructive control, vibration analysis, wavelet analysis.

\section{INTRODUCTION}

The feature of rolling bearing destruction is that it occurs suddenly, without the appearance of significant signs preceding the destruction, which entails unplanned labor costs associated with emergency stops, the malfunctions in the operation of equipment, and high repair costs. The solution of bearing early diagnosis problem will solve the problem of reasonable forecasting concerning the periods of trouble-free operation of equipment and the assignment of their resource in accordance with an actual condition of the bearing units.

The state of a rolling bearing, the development of its defects during the entire period of its service can be divided into five stages. Before the beginning of the first phase, the overall technical condition of a bearing is an ideal one. At the first stage, a defect appears and begins to develop in a bearing, and shock vibration impulses appear that grow in size. At the second stage, the shock impulses in a bearing reach almost their maximum energy. The quantitative value of the maximum pulse energy is determined by the type of a bearing and the conditions of its operation. At the third stage, the defect development zone is so large that the bearing begins to "lose" its main purpose - to ensure rotation of the shafts with minimal friction. The fourth stage is the last stage of defect development, when it covered the entire bearing. The fifth stage is the stage of waiting for an accident.

Due to the works by M.D. Genkin, A.G. Sokolova, F.Ya. Balitsky [1-3] significant advances have been achieved in the field of vibration control of machines, the analysis of which allows to detect a developing malfunction quickly, to assess its significance and to take measures to prevent an unplanned shutdown of the production process. The most significant studies in the field of vibration acoustic diagnostics of rolling bearings are given in the works by A.V. Frolova, A.V. Barkova, N.A. Barkova and V.A. Rusova [4-6]. Virtually all known vibration acoustic methods of control are based on the analysis of a signal itself or on the analysis of its frequency characteristics. Usually vibration of a bearing is recorded by a vibration sensor mounted on the bearing housing, where the sensor additionally collects the signals from other 
mechanical components - vibration sources. The operation of a bearing in the structure of a mechanism assumes the signal with a high noise level, thus the vibration characteristic of a bearing is distributed over a wide frequency band, on which noise and low-frequency effects are superimposed.

The analysis of literature data showed that vibration diagnosticians expect the greatest reliability and the greatest effect when the diagnosis of rolling bearings is introduced according to the spectra of vibrating signals. In this case, the errors of early diagnoses are predicted in advance. According to definition a spectrum is the power distribution of an initial time vibration signal in the frequency domain. Therefore, the appearance of pronounced narrow peaks on a spectrum in the zone of characteristic frequencies of a bearing element, which have a large amplitude and also a significant power, should be expected only if a defect develops to such an extent that its power will be commensurable with the power of clearly diagnosed peaks in a spectrum. In other words, a defect must be sufficiently developed, then it will be visible on the spectrum.

\section{METHODS}

The vibration generated by rolling bearing, in addition to the rotation speed $f_{k p}$ is characterized by the following fundamental frequencies [1]:

1. The frequency of rolling body rolling along an outer ring $f_{u}$ (1) (the presence of this component of vibration is determined by the fact that the rolling elements are not the best road through which the rotor shaft rolls, i.e., the shaft "jumps" on each rolling body):

$$
f_{H}=f_{c} \cdot Z=\frac{1}{2} f_{\theta p}\left(1-\frac{d_{m \kappa}}{d_{c}} \operatorname{Cos} \alpha\right) \cdot Z \approx 0,4 f_{\theta p} Z
$$

where $f_{c}$ is separator rotation frequency, $f_{b p}$ rotor rotation frequency, $d_{c} \approx\left(d_{H}+d_{b}\right) / 2$ - the separator diameter, i.e. the diameter of a circle passing through the centers of rolling bodies; here $d_{H}$ is the outer diameter of a bearing; $d_{B}$ is the inner diameter of a bearing; $d_{m \kappa}$ is a rolling element diameter; $\alpha$ - the contact angle of rolling elements with rolling ways; $\mathrm{Z}$ - the number of rolling bodies.

2. The rolling frequency of the rolling elements along the inner ring $f_{B}$ (2) (this vibration component appears if a shaft (an inner bearing ring) is not perfectly round, but, for example, 
has a local wear). Then the shaft "fails" on each rolling element when the latter falls into the wear zone:

$$
f_{\theta}=\left(f_{\theta p}-f_{c}\right) \cdot Z=\frac{1}{2} f_{в p}\left(1+\frac{d_{m \kappa}}{d_{c}} \operatorname{Cos} \alpha\right) \cdot Z \approx 0,6 f_{6 p} Z
$$

Always: $f_{u}+f_{\varepsilon}=Z f_{{ }_{p}}$.

3. The frequency of the separator (3) rotation (this component of vibration appears if one of the rolling bodies has a smaller (larger) diameter. Then the shaft "falls" or "bounces" when this body is under it).

$$
f_{c}=\frac{1}{2} f_{c p}\left(1-\frac{d_{m \kappa}}{d_{c}} \operatorname{Cos} \alpha\right)=\frac{1}{Z} f_{H} \approx 0,4 f_{\theta p}
$$

4. If a rolling element is not round, but has faces, then the shaft either "bounces" or "falls" with the frequency of its rotation $f_{m \kappa}(4)$.

$$
f_{m \kappa}=\frac{1}{2} f_{k p} \frac{d_{c}}{d_{m \kappa}}\left(1-\frac{d_{m \kappa}^{2}}{d_{c}^{2}} \operatorname{Cos}^{2} \alpha\right)
$$

The signal of a defect-free bearing has two frequencies: the rotor speed $f_{g p}$ and the rolling frequency of rolling elements along an outer ring $f_{u}$. The frequency amplitude $f_{u}$ is two times less than the frequency amplitude $f_{6 p}$.

In order to study in the Lab View environment the program was developed to simulate rolling bearing signals. It generated the signals of defect-free bearings and the bearings with different types of defects of 207 series at the rotation speed of a bearing inner ring making 2400 . The simulated signals of defective bearings have four frequencies:

- Rotor rotation frequency $f_{6 p}$;

- The frequency of rolling element rolling along the outer ring $f_{H}$;

- Separator rotation frequency $f_{c}$;

- The frequency of rolling element rolling along the inner ring $f_{6}$.

Since in real conditions the rolling bearings in rotary machines are the source of bearing vibrations with a wide spectrum of frequencies (from several $\mathrm{Hz}$ to tens of $\mathrm{kHz}$ ), close in nature to stochastic ones, several types of noise were additionally added to each signal $[7,8]$ :

- Poisson noise, a pseudo-random sequence of values is generated that represents the number of events of the ordinary Poisson process appearing on a given interval; 
- Uniform white noise, generates a pseudo-random white noise whose spectral components are evenly distributed over the entire range of involved frequencies

- Periodic random noise generated and its output array contains all the frequencies that can be represented by an integer number of periods on a given interval, each frequency component has a random phase, so an output array can be represented as the result of sinusoid summation with identical amplitudes and random phases bounded in magnitude by the following values::

амплитуда_спектра $\cdot\left(\frac{\text { выборки }}{2}-1\right)$, if the samples are an even number;

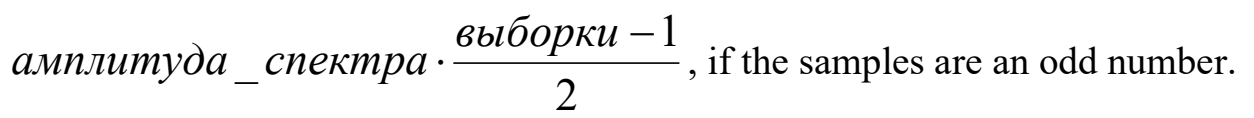

Two groups of signals were modeled with different amplitudes of noise frequency components:

- The amplitude of noise frequency components is ten times smaller than the amplitude of the rotor speed $f_{s p}(\mathrm{a})$, the defects in equipment with low noise level or the defects at a late stage of development

- The amplitude of noise frequency components is commensurate with the amplitude of the rotor speed $f_{6 p}$ (б), the defects in equipment with a high level of noise or the defects at an early stage of development.

The example of simulated signals, their spectra and scale graphs of continuous wavelet analysis, using the Mexican hat wavelet as the basis, with the amplitude of the noise component below the amplitude of the defect frequencies a) are shown on Fig. 1 and above the amplitude of the defect frequencies b) on Fig. 2.

The wavelet Mexican hat [9] is calculated from the second derivative $(n=2)$ and is shown on Figure 3. The wavelet is symmetric, the wavelet spectrum is represented only by the real part and is well localized by frequency, zero and the first moments of the wavelet are zero. It is used to analyze complex signals. The wavelet equation:

$$
\psi(t, a, b)=\frac{1.031}{\sqrt{2}} \cdot\left\{\exp \left[-\left(\frac{t-b}{a}\right)^{2}\right]-2\left(\frac{t-b}{a}\right)^{2} \cdot \exp \left[-\left(\frac{t-b}{a}\right)^{2}\right\} .\right.
$$



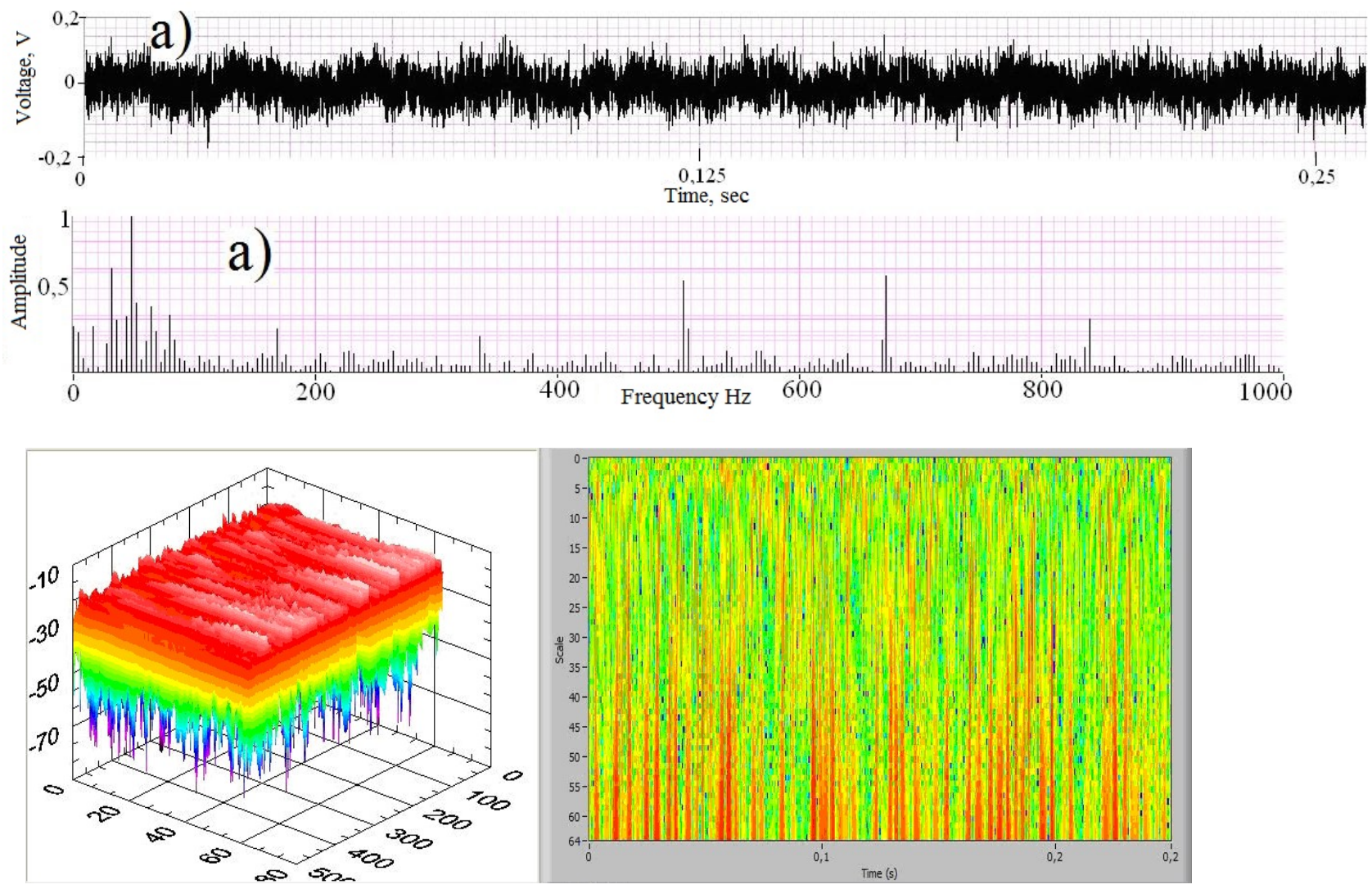

Fig.1. Signal, spectrum and wavelet scaling graph of a defect-free bearing with low amplitudes of frequency components 

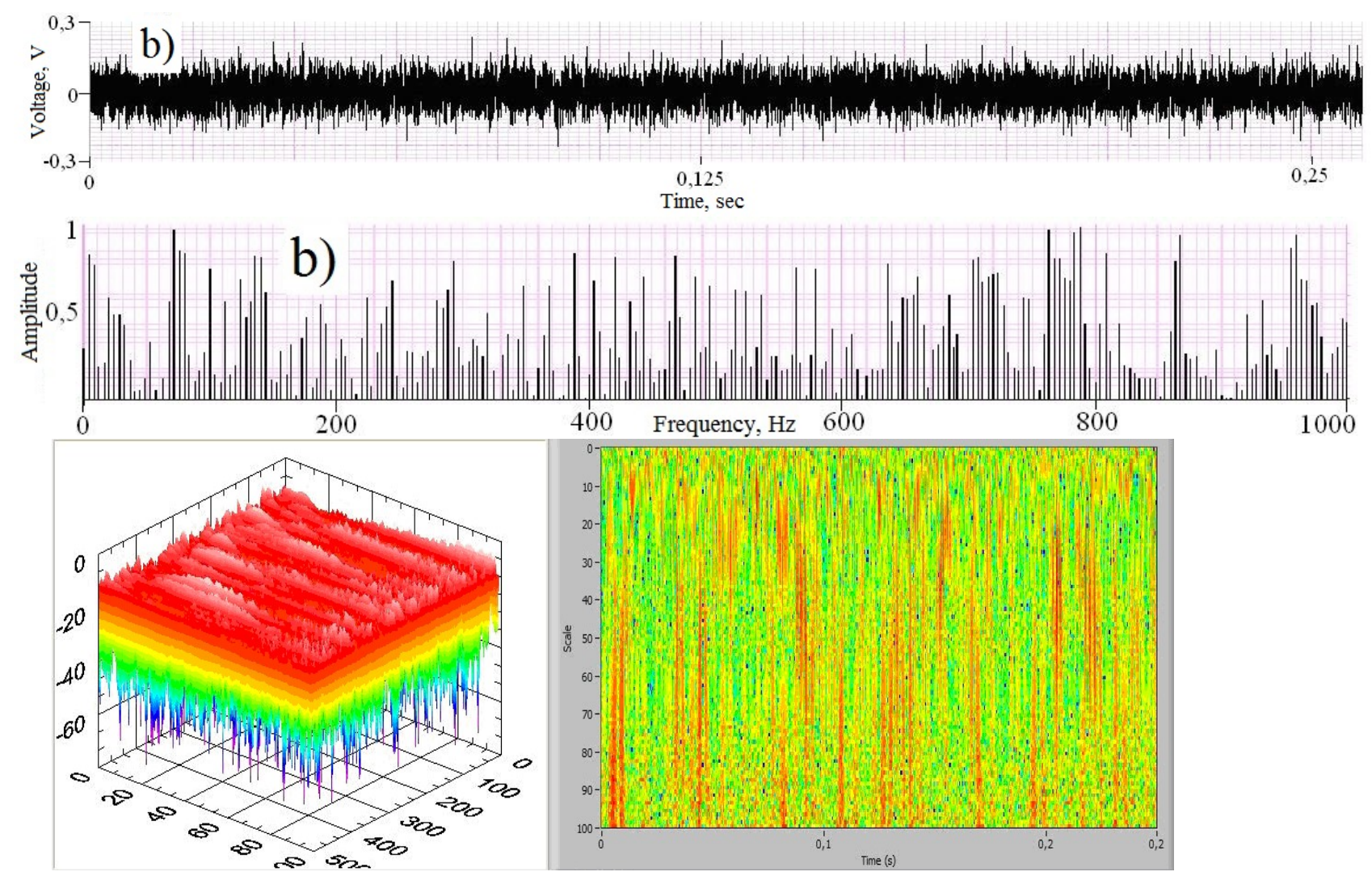

Fig.2. Signal, spectrum and wavelet scaling of a defect-free bearing with high amplitude of frequency components
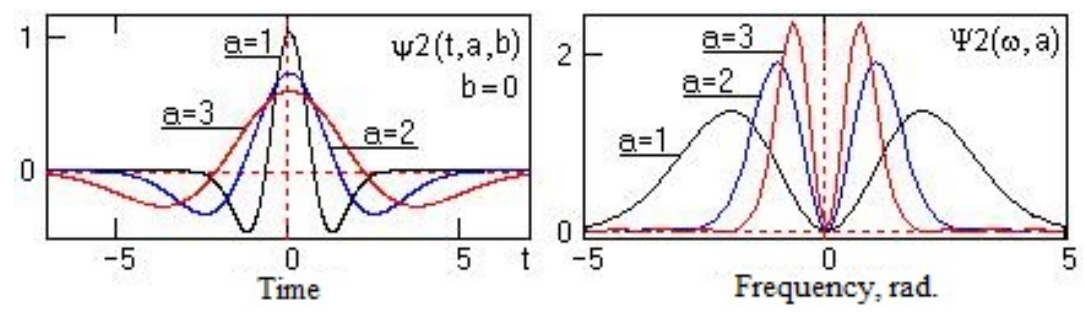

Fig.3. Mexican hat wavelet

The description of bearing defects and their corresponding simulated signals are presented in Table 1. And the noises have been superimposed on the simulated signals for a defect-free bearing.

\section{RESULTS}

Using the "DetectFault" program [10], the spectra of the simulated signals were compared by the correlation coefficient $r$ of the spectra and by the Spearman's rank correlation coefficient. 
The following relationship is used to calculate the selective correlation coefficient $r$ :

$$
r=\frac{\sum \mathrm{a}_{i} \mathrm{a}_{s i}-\left(\sum \mathrm{a}_{i} \sum \mathrm{a}_{s i}\right) / n}{\sqrt{\left(\sum \mathrm{a}_{i}^{2}-\left(\sum \mathrm{a}_{i}\right)^{2} / n\right) \cdot\left(\sum \mathrm{a}_{s i}^{2}-\left(\sum \mathrm{a}_{s i}\right)^{2} / n\right)}}
$$

A nonparametric rank estimate of the Spearman correlation rs was calculated according to the expression (7):

$$
r_{s}=1-\frac{6}{n\left(n^{2}-1\right)} \sum_{i=1}^{n}\left(\operatorname{rank} a_{i}-\operatorname{rank} a_{s i}\right)^{2}
$$

where rank $a_{\mathrm{i}}$ is the rank of the amplitude $a_{\mathrm{i}}$ in the variational series of the tested spectrum amplitudes (that is, the number of the place that this amplitude occupies among all the amplitudes of the given spectrum, ordered in ascending order); rank $a_{\mathrm{si}}$ - the same for the reference spectrum.

\begin{tabular}{|c|c|c|c|c|}
\hline $\begin{array}{l}\text { Bearing } \\
\text { № }\end{array}$ & $\begin{array}{l}\text { Modeled } \\
\text { frequency }\end{array}$ & $\begin{array}{c}\text { Frequency } \\
\text { amplitude value }\end{array}$ & $\begin{array}{l}\text { Additional } \\
\text { harmonics }\end{array}$ & Modeled defect description \\
\hline 1,2 & $\begin{array}{l}f_{\theta p} \\
f_{H}\end{array}$ & $\begin{array}{c}1 \\
0,5\end{array}$ & ---- & ---- \\
\hline 3,4 & $\begin{array}{l}f_{6 p} \\
f_{H} \\
f_{c} \\
f_{6}\end{array}$ & $\begin{array}{c}1 \\
0,5 \\
0,5 \\
0,5\end{array}$ & ---- & $\begin{array}{l}\text { the beginning of the second } \\
\text { stage of defect development } \\
\text { of an outer bearing ring (the } \\
\text { scoring is more than } 0.1 \\
\text { mm) }\end{array}$ \\
\hline 5,6 & $\begin{array}{l}f_{6 p} \\
f_{H} \\
f_{c} \\
f_{6}\end{array}$ & $\begin{array}{l}1 \\
2 \\
2 \\
2\end{array}$ & ---- & $\begin{array}{l}\text { the beginning of the third } \\
\text { stage of an outer ring defect } \\
\text { development (crack) }\end{array}$ \\
\hline 7,8 & $f_{\theta p}$ & 1 & ---- & the second stage of rolling \\
\hline
\end{tabular}

Table 1. Characteristics of bearing signals 


\begin{tabular}{|c|c|c|c|c|}
\hline & $\begin{array}{l}f_{n} \\
f_{c} \\
f_{6}\end{array}$ & $\begin{array}{c}0,5 \\
1 \\
0,5\end{array}$ & & $\begin{array}{l}\text { ball defect development (the } \\
\text { scoring is more than } 0.1 \\
\mathrm{~mm} \text { ) }\end{array}$ \\
\hline 9,10 & $\begin{array}{l}f_{6 p} \\
f_{u} \\
f_{c} \\
f_{6}\end{array}$ & $\begin{array}{c}1 \\
0,5 \\
0,5 \\
1\end{array}$ & $\begin{array}{l}\text { Six harmonics of } \\
\text { frequency } f_{\sigma}\end{array}$ & $\begin{array}{l}\text { the third stage of rolling ball } \\
\text { defect development (the } \\
\text { chip is more than } 0.2 \mathrm{~mm} \text { ) }\end{array}$ \\
\hline 11,12 & $\begin{array}{l}f_{\theta p} \\
f_{n} \\
f_{c} \\
f_{b}\end{array}$ & $\begin{array}{c}1 \\
0,5 \\
0,5 \\
0,5\end{array}$ & ---- & $\begin{array}{l}\text { the second stage of the } \\
\text { bearing inner ring defect } \\
\text { development (the scoring is } \\
\text { more than } 0.1 \mathrm{~mm} \text { ) }\end{array}$ \\
\hline 13,14 & $\begin{array}{l}f_{c p} \\
f_{u} \\
f_{c} \\
f_{6}\end{array}$ & $\begin{array}{c}1 \\
1 \\
0,5 \\
0,5\end{array}$ & $\begin{array}{l}\text { Six harmonics of } \\
\text { frequency } f_{H}\end{array}$ & $\begin{array}{l}\text { the third stage of the inner } \\
\text { bearing ring defect } \\
\text { development (crack) }\end{array}$ \\
\hline
\end{tabular}

In order to classify the bearings into defect-free or defective class (Figure 4), the approach is proposed that is used to control products by the method of free oscillations [11-14] characteristic of the procedures for anomaly rejection: the analysis algorithm interprets a set of computed values of some statistics $\left(\mathrm{p}_{1}, \mathrm{p}_{2}, \ldots, \mathrm{p}_{\mathrm{m}}\right)$ as a set of measured values of an abstract parameter and applies the following procedure to this set:

1) the position estimate $p$ is calculated;

2) the estimate of the spread $\mathrm{S}$ is calculated as the median of absolute deviations with respect to the position estimate

$$
S=\frac{\sum_{i=1}^{m}\left|p_{i}-\bar{p}\right|}{m}
$$


3) a confidence interval is developed for a given level of significance $\alpha$

$$
\bar{p} \pm S \cdot t\left(1-\frac{\alpha}{2}, m-2\right),
$$

where $t(\alpha, m)-\alpha$-quantile of Student's distribution with $\mathrm{m}$ degrees of freedom.

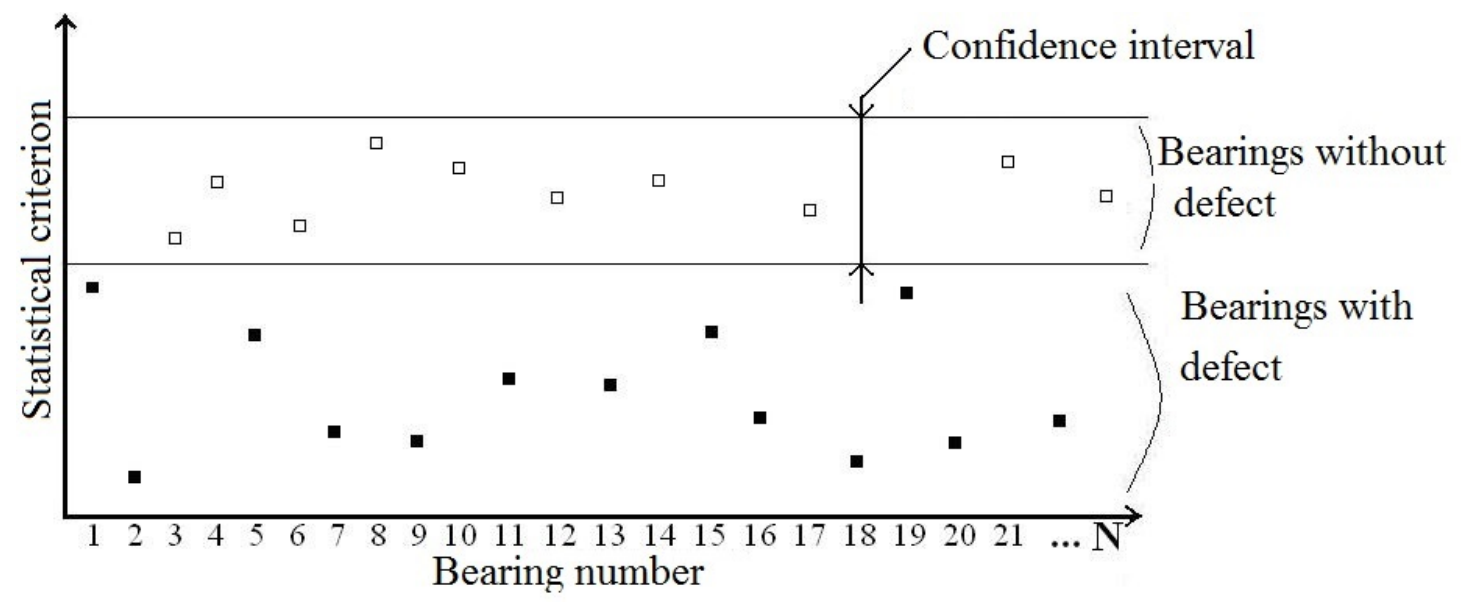

Fig.4. Classification of bearings: defect-free or defective

After the processing it was established that the results of spectrum comparison by Spearman's rank correlation are similar to the results of the comparison by spectrum correlation coefficient. As an example, Figure 5 provides five values of comparison results concerning the spectra according to the correlation coefficient of the simulated signals (Table 1) for defect-free (bearing number 1-2) and defective rolling bearings (3-14) with the amplitude of the noise component below a) and above b) defect frequency amplitude. 

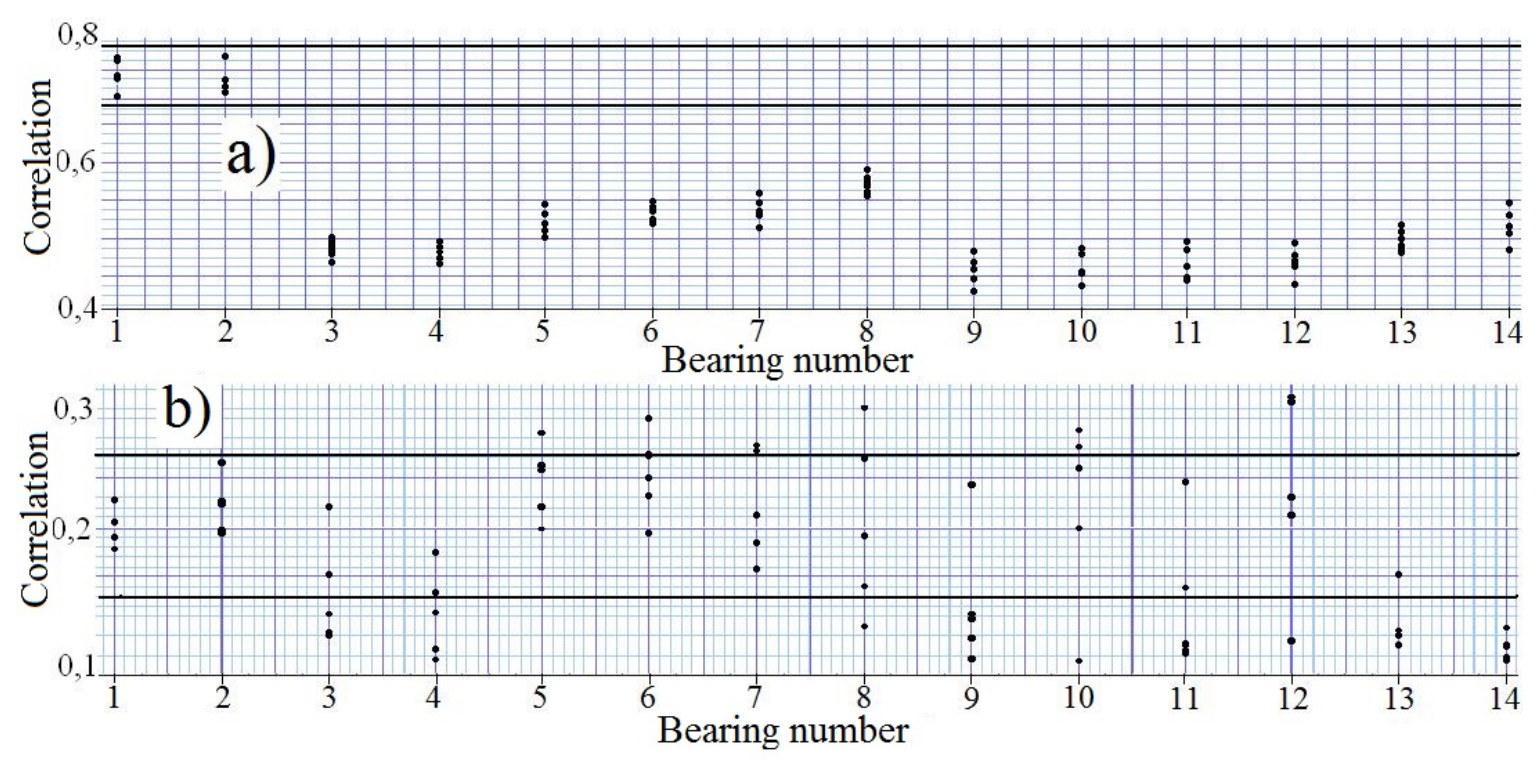

Fig.5. Simulated signal spectrum comparison results

After the analysis of the data presented on Figure 5 they revealed that static criteria for spectrum comparison allow to detect the defects at the noise levels much lower than the level of the bearing signal or at a late stage of defect development and are not applicable for early diagnosis of rolling bearings or the rolling bearings in equipment where the noise level exceeds the level of the bearing signal.

The comparison of signal scale graphs by PSNR metrics was performed based on forty types of wavelet basis, such as Meyer, Morlet, MexicanHat, Haar, db02, db03, db04, db05, db06, db07, db08, db09, db10, db11, db12, db13, db14, sym2, sym3, sym4, sym5, sym6, sym7, sym8, coif1, coif2, coif3, coif4, coif5, bior1_3, bior1_5, bior2_2, bior2_4, bior2_6, bior2_8, bior3_1, bior3_3, bior3_5, bior3_7, bior3_9 (Table 2) [12, 13].

The abbreviation PSNR (peak signal-to-noise ratio) is the term meaning the ratio between the maximum of the possible signal value and the noise power distorting the signal values and is calculated by the following formula:

$$
P S N R=20 \times \log \left(\frac{\text { Max_value }}{\sqrt{\frac{\prod_{i=0}^{\min \left(b^{s}, b\right)} \square_{j=f_{1}}^{f_{2}}\left(C_{i}^{s}[j]-C_{i}[j]\right)^{2}}{\min \left(b^{s}, b\right)}}}\right)
$$


where Max_value is the amplitude of the maximum possible power signal allowed in this representation of an audio signal; $C_{i}^{S}[j]$ and $C_{i}[j]$ - the amplitude values of the scaling graphs concerning a reference and an examined bearing; $b^{s}$ and $b-$ is the number of wavelet basis shifts for the first and thesecond signal, respectively; $f_{1}$ and $f_{2}$ - the parameters that filter out the frequency band for the study from the general scaling graph. The advantage of PSNR metrics use is that it allows you to consider changes in individual frequency ranges and, at the same time, it is not sensitive to a signal phase.

\section{DISCUSSION}

Figure 6 provides five values of the comparison results concerning the wavelet scaling graphs of the simulated signals are presented (Table 1) with a standard scaling graph, the PSNR metrics, Mexican hat as wavelet basis, defect-free (bearing number 1-2) and defective rolling bearings (3-14) with the amplitude of the noise component below a) and above b) the amplitude of defect frequencies.

Table 2. Basic wavelet functions

\begin{tabular}{|l|l|l|}
\hline Mnemonic designation & \multicolumn{1}{|c|}{ Wavelet name } & Corresponding wavelet characteristics \\
\hline Meyer & Meyer's wavelet & Wavelet with an infinite smoothness \\
\hline Morlet & Marlet's wavelet & \multirow{2}{*}{ Wavelet with an infinite carrier } \\
\cline { 1 - 2 } Mexican Hat & "Mexican hat" wavelet & \\
\hline Haar & Haar's wavelet & \\
\hline Db№ & Daubechy's wavelet & \multirow{2}{*}{ Orthogonal wavelets with a finite mask } \\
\cline { 1 - 2 } Sym№ & "Simlets" & \\
\hline Coif№ & "Coiflets" & \\
\hline Bior№ & Biorthogonal wavelet & Biorthogonal wavelets with a finite mask \\
\hline
\end{tabular}



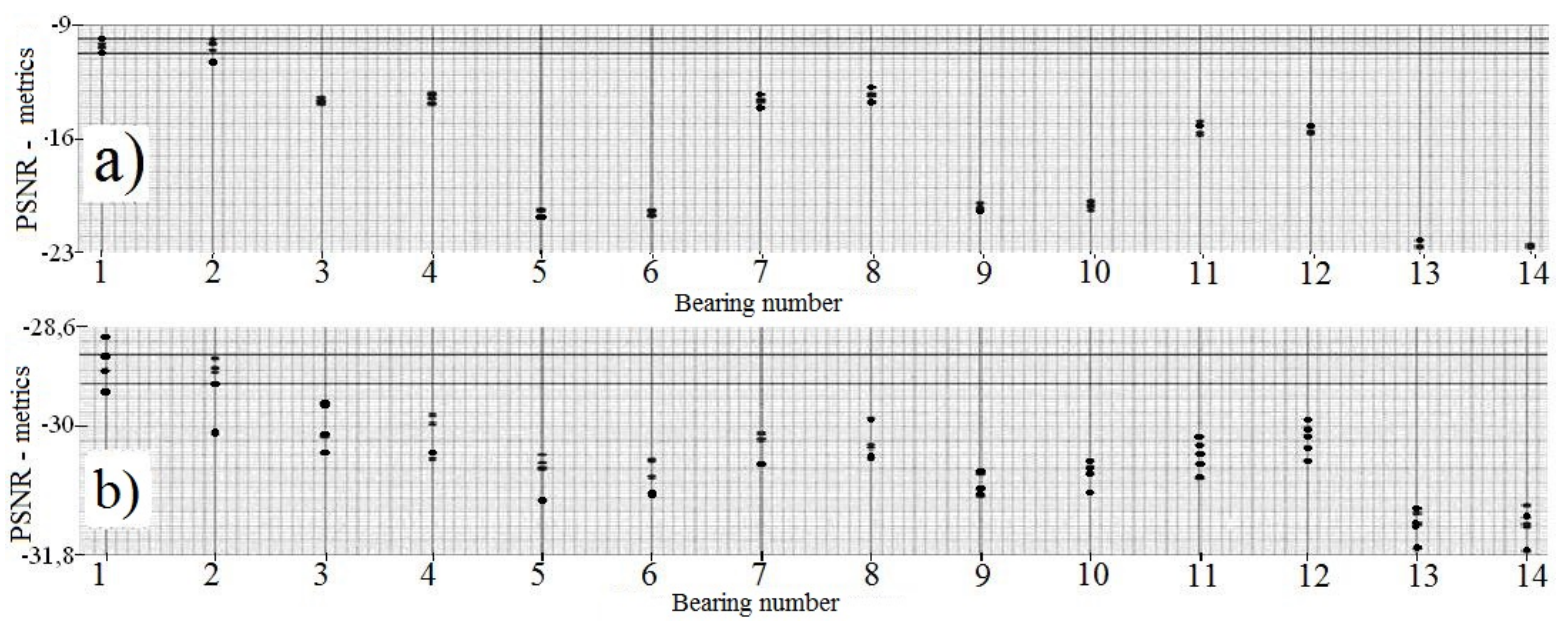

Fig.6. Comparison results of scaling graphs for simulated PSNR signals by metric, basis wavelet type - Mexican hat

\section{CONCLUSIONS}

The analysis of the obtained data showed the following:

- the criteria for spectrum comparison allow the detection of defects at the noise levels much lower than the bearing signal level or at a late stage of defect development and are not applicable to early diagnosis of rolling bearings or the rolling bearings in the equipment where the noise level exceeds the bearing signal level;

- all the above-mentioned basic wavelet functions are suitable for bearing control, regardless of a defect development stage and the noise level.

- Since each defect has different frequencies, and the PSNR metric allows one to consider the differences in separate frequency ranges, it can also be used to classify a defect at an early stage of development.

\section{SUMMARY}

Continuous wavelet transformation and PSNR metric, as a comparison criterion, are applicable for vibration diagnostics of rolling bearings and have a number of advantages over classical spectral methods of signal processing: they allow to detect defects at an early stage of nucleation; the determination of a defect depends little on the degree of noisiness, in the case of spectrum comparison with a noise component increase by 10 times, the determination of a defect is impossible, and during the comparison of scaling graphs the probability of a defect detection is reduced by 1.2 times. 
It is necessary to create an apparatus to measure the vibration level of rolling bearings and to carry out experimental studies thereon for further study of continuous wavelet transformation and PSNR metrics possibility to analyze the scaling graphs of the vibration-acoustic signal for rolling bearings.

\section{ACKNOWLEDGEMENTS}

The work is performed according to the Russian Government Program of Competitive Growth of Kazan Federal University.

\section{REFERENCES}

[1] Genkin M.D., Sokolova A.G. Vibration-acoustic diagnostics of machines and mechanisms. M: mechanical engineering, 1987.

[2] Balitsky F.Ya., Genkin M.D., Ivanova M.A., Sokolova A.G., Khomyakov E.I. Vibration-acoustic diagnosis of incipient defects. Moscow: Nauka, 1984.

[3] Balitsky F.Ya., Genkin M.D., Ivanova M.A., Sokolova A.G., Khomyakov E.I. Modern methods and the means of vibration diagnostics of machines and structures. Moscow: MTSNTI, 1990.

[4] Barkov A, Barkova N. Condition Assessment and Life Prediction of Rolling Element Bearing. «Sound and Vibration», USA, part 1, 06, 1995.

[5] Barkov A, Barkova N. Condition Assessment and Life Prediction of Rolling Element Bearing. «Sound and Vibration», USA, part 2, 09, 1995.

[6] Barkov A.V., Barkova N.A. Intelligent monitoring and diagnostics systems of machines by vibration. Proceedings of Petersburg energy institute of training executives and experts of the Ministry of Energy of the Russian Federation and USA vibration Institute, vol. 9, 1999.

[7] Vibrations in machinery: A handbook. 6 volumes. Ed. board: V.N. Chelomei (chairman) - M: Mechanical Engineering, 1978.

[8] Zverev V.A., Stromkov A.A. The approximation of signals from interference by numerical methods, 2001, $187 \mathrm{p}$.

[9] Goswami J.C., Chan A.K. Fundamentals of Wavelets: Theory, Algorithms, and Applications, 1999

[10] Akutin M.V., Vankov Yu.V., Busarov A.V., Ziganshin Sh.G. Certificate of state registration for the computer program DetectFault, № 2008610104, application number $2007614295,2007$. 
[11] Saifullin E.R., Vankov Y.V., Busarov A.V. Inspection of riveted connections by free oscillation technique. Inspection of riveted connections for "riveted wheel blades" at the production stage (2016) International Journal of Pharmacy and Technology, 8 (4), pp. 2438424399

[12] Saifullin E.R., Vankov Y.V., Busarov A.V. Control for riveted connections by free oscillation technique. Hardware and software system. Evaluation of measurement error (2016) International Journal of Pharmacy and Technology, 8 (4), pp. 24343-24356

[13] Saifullin, E.R., Vankov, Y.V., Busarov, A.V.Testing method for riveted connections using the free oscillation technique. Numerical simulation (2016) International Journal of Pharmacy and Technology, 8 (4), pp. 24231-24241

[14] Yu V Vankov, Sh G Ziganshin, E V Izmailova, A R Zagretdinov The effect of the size and position of transverse cracks in the natural frequencies of the rods IOP Conference Series: Materials Science and Engineering 2015 DOI: 10.1088/1757-899X/86/1/012036

[15] Dobeshy I. Ten lectures on wavelets. Publ. Regular and chaotic dynamics. 2001, 464 p.

[16] Malla S. Wavelets in signal processing (Wavelet Tour of Signal Processing).

Publishing house "Mir", 2005, 672 p.

\section{How to cite this article:}

Rinatovich S E, Vitalevich V Y, Victorovich A M, Vyacheslavovna I E. Rolling bearing monitoring algorithm by wavelet scaling comparison. J. Fundam. Appl. Sci., 2017, 9(2S), 957-971. 